\title{
@creative
}

\section{The modified Wiener index of some graph operations}

\author{
Ali Reza Ashrafi, Hossein Shabani \\ Department of Pure Mathematics, Faculty of Mathematical Sciences, \\ University of Kashan, Kashan 87317-53153, I. R. Iran
}

Received 20 January 2015, accepted 22 August 2015, published online 16 December 2015

\begin{abstract}
Graovac and Pisanski [On the Wiener index of a graph, J. Math. Chem. 8 (1991) 53 62] applied an algebraic approach to generalize the Wiener index by symmetry group of the molecular graph under consideration. In this paper, exact formulas for this graph invariant under some graph operations are presented.
\end{abstract}

Keywords: Modified Wiener index, graph operation, automorphism group.

Math. Subj. Class.: 20C15, 20D15

\section{Introduction}

Throughout this paper graph means simple connected graphs. The distance between the vertices $u, v$ of a graph $G, d_{G}(u, v)$ (or $d(u, v)$ for short), is defined as the number of edges in a shortest path connecting them. The sum of all distances between vertices in $G$ is called the Wiener index of $G$ [9]. The first study of this number were made by Harold Wiener in 1947 who realized that there are correlations between this graph invariant and the boiling points of paraffin. We encourage the reader to consult $[1,2]$ and references therein for information about the effect of this graph invariant on trees and hexagonal systems and [4] for some applications in chemistry.

Let $G=(V, E)$ be a simple graph with the vertex set $V$ and the edge set $E$. Graovac and Pisanski [3] in a seminal paper applied the symmetry group of the graph under consideration to generalize the Wiener index. To explain, we assume that $\Gamma$ is the automorphism group of $G$. Then the distance number of an automorphism $g, \delta(g)$, is defined as the average of $d(u, g(u))$ over all vertices $u \in V(G)$ and

$$
\delta(G)=\frac{1}{|\Gamma|} \sum_{g \in \Gamma} \delta(g)=\frac{1}{|\Gamma||V(G)|} \sum_{u \in V(G)} \sum_{g \in \Gamma} d(u, g(u)) .
$$

E-mail addresses: ashrafi@kashanu.ac.ir (Ali Reza Ashrafi), shabani@grad.kashanu.ac.ir (Hossein Shabani) 
Define:

$$
\widehat{W}(G)=\frac{|V(G)|}{2|\Gamma|} \sum_{u \in V(G)} \sum_{g \in \Gamma} d(u, g(u)) .
$$

It can be easily shown that

$$
\widehat{W}(G)=\frac{1}{2}|V(G)|^{2} \delta(G) .
$$

The authors of [3], in their pioneering work used the name "modified Wiener index" for this graph invariant. Suppose $e=u v \in E(G)$ and $V(e)=\{u, v\}$. The line graph $L(G)$ is a graph with $E(G)$ as vertex set. Two different vertices of $V(L(G))$ are adjacent if and only if they have a common vertex in $G$. The subdivision graph $S(G)$ is the graph obtained by inserting an additional vertex in each edge of $G$. In other words, each edge of the subdivision graph is replaced by a path of length 2 .

Suppose $G$ is a graph. Following Yan et al. [8], we set $E E(G)=\{\{e, f\} \mid e, f \in$ $E(G) \&|V(e) \cap V(f)|=1\}$ and $E V(G)=\{\{e, v\} \mid v \in V(G) \& v \in V(e) \& e \in$ $E(G)\}$, where $V(e)$ is the set of all end vertices of the edge $e$. Define the line graph $L(G)$, the subdivision graph $S(G)$, the total graph $T(G)$ and the graphs $R(G)$ and $Q(G)$ as follows:

$$
\begin{array}{llrl}
V(L(G)) & =E(G), & & E(L(G))=E E(G), \\
V(S(G)) & =V(G) \cup E(G), & & E(S(G))=E V(G), \\
V(T(G)) & =V(G) \cup E(G), & & E(T(G))=E(G) \cup E V(G) \cup E E(G), \\
V(R(G)) & =V(G) \cup E(G), & & E(R(G))=E(G) \cup E V(G), \\
V(Q(G)) & =V(G) \cup E(G), & & E(Q(G))=E V(G) \cup E E(G) .
\end{array}
$$

Throughout this paper we use the standard notations of group theory and graph theory. We refer to [5], for the main properties of product graphs. If $G$ is a connected graph then the diameter $d=\operatorname{diam}(G)$ is defied as the length of the largest distance between two vertices in $G$. Moreover, define

$$
\bar{D}(g, i)=\left|\left\{\{u, g(u)\} \mid u \in V(G) \& d_{G}(u, g(u))=i\right\}\right| ; 1 \leq i \leq d,
$$

and $\bar{D}(\Gamma, i)=\sum_{g \in \Gamma} \bar{D}(g, i) ; 1 \leq i \leq d$. The number of $\{u, v\}$ such that $d(u, v)=i$ in $\widehat{W}(G)$ is equal to $\bar{D}(G, i)=\bar{D}(\Gamma, i)$. Suppose $x$ and $y$ are vertices of $G$. We write $x \sim_{G} y$ to show that $x, y$ are adjacent in $G$. They are called equivalent, $x \approx_{G} y$, if there exists an automorphism $\alpha$ such that $\alpha(x)=y$. The path, cycle and complete graphs with $n$ vertices are denoted by $P_{n}, C_{n}$ and $K_{n}$, respectively. The number of edges in a path $P$ is denoted by $l(P)$ and named the length of $P$. Our other notations are standard and taken mainly from the standard books on these topics.

Main Theorem. Suppose $G$ is a tree of diameter $d$. Then the following relations hold:

1. $\widehat{W}(L(G))=\frac{n-1}{n} \widehat{W}(G)-\frac{n-1}{2|\Gamma|} \sum_{i=1}^{d} \bar{D}(G, i)$,

2. $\widehat{W}(S(G))=\frac{4 n-2}{n} \widehat{W}(G)+\frac{4 n-2}{n-1} \widehat{W}(L(G))$,

3. $\widehat{W}(T(G))=\frac{2 n-1}{n} \widehat{W}(G)+\frac{2 n-1}{n-1} \widehat{W}(L(G))$, 
4. $\widehat{W}(Q(G))=\frac{4 n-2}{n} \widehat{W}(G)$,

5. $\widehat{W}(R(G))>\frac{4 n-2}{n} \frac{|\Gamma|}{|\operatorname{Aut}(R(G))|} \widehat{W}(G)$.

\section{Proof of the Main Theorem}

In [6], a character theoretical method for computing the modified Wiener index of graphs is presented and in [8], the authors computed exact formulas for the Wiener index under five graph operations. The aim of this paper is to continue these papers by computing the modified Wiener index of trees under the graph operations $L(-), S(-), T(-), Q(-)$ and $R(-)$. For simplicity of our argument, we assume that $\Gamma=\operatorname{Aut}(G)$ and $\bar{W}(G)=$ $\frac{2|\Gamma|}{|G|} \widehat{W}(G)$ then $\bar{W}(G)=\sum_{i=1}^{d} i \cdot \bar{D}(G, i)$. We will start by stating a well-known result in algebraic graph theory.

Lemma 2.1. Suppose $G$ is a tree with at least three vertices. Then $\operatorname{Aut}(L(G)) \cong \operatorname{Aut}(G)$.

Proof. It is an immediate consequence of [7, Corollary 1.4].

Theorem 2.2. Let $G$ be a tree with $n \geq 3$ vertices and $\Gamma=\operatorname{Aut}(G)$. Then, $\widehat{W}(L(G))=$ $\frac{n-1}{n} \widehat{W}(G)-\frac{n-1}{2|\Gamma|} \sum_{i=1}^{d} \bar{D}(G, i)$.

Proof. Suppose $e=u v$ and $f=x y$ are vertices of $L(G)$ such that $e \approx_{L(G)} f$ and $d_{L(G)}(e, f)=i$. So, there are $\sigma \in \operatorname{Aut}(G)$ and $\bar{\sigma} \in \operatorname{Aut}(L(G))$ such that $\bar{\sigma}(e)=f$, $\sigma(u)=x$ and $\sigma(v)=y$. Choose a shortest path $e=e_{0}, e_{1}, \ldots, e_{i}=f$ in $L(G)$. Set $e=e_{0}=u_{0} u_{1}, e_{1}=u_{1} u_{2}, \ldots, e_{i-1}=u_{i-1} u_{i}, f=e_{i}=u_{i} u_{i+1}, u=u_{0}, v=u_{1}$, $y=u_{i}$ and $x=u_{i+1}$. Since $G$ is a tree, $u=u_{0}, u_{1}, \ldots, u_{i}, u_{i+1}=x$ is a shortest path in $G$ connecting $u=u_{0}$ and $x=u_{i+1}$. Thus $d_{G}(u, x)=i+1$. Therefore, for any vertices $e$ and $f$ of $L(G)$ at distance $i$, we fined two vertices $u_{e}$ and $u_{f}$ of $G$ at distance $i+1$, corresponding to $e$ and $f$, respectively.

We now assume that $r$ and $s$ are vertices in $G$ at distance $i$ and $r=v_{0}, v_{1}, \ldots, v_{i-1}$, $v_{i}=s$ is the unique shortest path connecting $r$ and $s$. Then the edges $v_{0} v_{1}$ and $v_{i-1} v_{i}$ are at distance $i-1$ in $L(G)$. Hence, $\bar{D}(L(G), i)=\bar{D}(G, i+1)$. Therefore,

$$
\begin{aligned}
\bar{W}(L(G)) & =\sum_{i} i \bar{D}(L(G), i) \\
& =\sum_{i}(i-1) \bar{D}(G, i) \\
& =\sum_{i} i \bar{D}(G, i)-\sum_{i} \bar{D}(G, i) \\
& =\bar{W}(G)-\sum_{i} \bar{D}(G, i) .
\end{aligned}
$$

Therefore, $\frac{2|\operatorname{Aut}(L(G))|}{|V(L(G))|} \widehat{W}(L(G))=\frac{2|\Gamma|}{|V(G)|} \widehat{W}(G)-\sum_{i} \bar{D}(G, i)$, which completes our argument.

Lemma 2.3. Suppose $G$ is a tree. Then $\operatorname{Aut}(S(G)) \cong \operatorname{Aut}(G)$.

Proof. Define $\Phi: \operatorname{Aut}(G) \longrightarrow \operatorname{Aut}(S(G))$ given by $\left.\Phi(\alpha)\right|_{V(G)}=\alpha$ and if $e=x y \in$ $E(G)$ then $\Phi(\alpha)(e)=\alpha(x) \alpha(y) \in E(G)$. Notice that if $x \sim_{G} y$ and $t, t^{\prime}$ are vertices 
in $S(G)$ such that $x \sim_{S(G)} t \sim_{S(G)} y$ and $\Phi(\alpha)(x) \sim_{S(G)} t^{\prime} \sim_{S(G)} \Phi(\alpha)(y)$ then $\Phi(\alpha)(t)=\Phi(\alpha)\left(t^{\prime}\right)$. It can easily be proved that $\Phi(\alpha)$ is a permutation of $S(G)$. We show that $a b \in E(S(G))$ if and only if $\Phi(\alpha)(a) \Phi(\alpha)(b) \in E(S(G))$. Suppose $a \sim_{S(G)} b$ and $a \in G$. Then there exists $c \in G$ such that $a \sim_{G} c$ and $a \sim_{S(G)} b \sim_{S(G)} c$. Since $\alpha \in \operatorname{Aut}(G), a c \in E(G)$ if and only if $\alpha(a) \alpha(c) \in E(G)$. Hence, there exists $l \in S(G)$ such that $\Phi(\alpha)(a) \sim_{S(G)} l \sim_{S(G)} \Phi(\alpha)(c)$. This implies that $\Phi(\alpha)(b)=l$. So, $a b \in$ $E(S(G))$ if and only if $\Phi(\alpha)(a) \Phi(\alpha)(b) \in E(S(G))$. Similarly, if $\bar{\sigma} \in \operatorname{Aut}(S(G))$ then $\sigma=\left.\bar{\sigma}\right|_{G} \in \operatorname{Aut}(G)$. Thus, $\Phi$ is invertible.

Theorem 2.4. Let $G$ be a tree with $n \geq 3$ vertices. Then

$$
\widehat{W}(S(G))=\frac{4 n-2}{n} \widehat{W}(G)+\frac{4 n-2}{n-1} \widehat{W}(L(G)) .
$$

Proof. Suppose $x, y \in V(S(G))$ are in the same orbit of $\operatorname{Aut}(S(G)), d_{S(G)}(x, y)=k$ and $\tau(x)=y$, where $\tau \in \operatorname{Aut}(S(G))$. It is obvious that both of $x$ and $y$ must be together in $V(G)$ or $E(G)$. We first assume that $x, y \in V(G)$. Choose the shortest path $P_{1}: x=$ $u_{0}, u_{1}, \ldots, u_{k}=y$ in $S(G)$. Obviously, if $i$ is even then $u_{i} \in G$ and so $k=2 k^{\prime}$. Since $G$ is tree, $P_{2}: x=u_{0}, u_{2}, \ldots, u_{k^{\prime}}=y$ is the unique path connecting $x$ and $y$ in $G$. Hence, $d_{S(G)}(x, y)=2 d_{G}(x, y)$.

Next we assume that $x, y \notin V(G), P_{3}: x=u_{0}, u_{1}, \ldots, u_{k}=y$ is a shortest path in $S(G)$. Choose edges $a b, c d \in E(G)$ such that $a \sim_{S(G)} x \sim_{S(G)} b$ and $c \sim_{S(G)} y \sim_{S(G)} d$. Suppose that $\tilde{x}$ and $\tilde{y}$ are corresponding vertices of $x$ and $y$ in $L(G)$, respectively. Since $S(G)$ is tree, the path $P_{3}$ is unique and so the vertices $u_{i}, i$ is even, are corresponding to vertices $\tilde{u}_{i}$ in $L(G)$. This proves that $k$ is even, say $k=2 k^{\prime}$. In a similar way, there exists a path $P_{2}: \tilde{x}=\tilde{u}_{0}, \tilde{u}_{1}, \ldots, \tilde{u}_{k^{\prime}}=\tilde{y}$ in $L(G)$. So, we have again $d_{S(G)}(x, y)=$ $2 d_{L(G)}(\tilde{x}, \tilde{y})$. Thus,

$$
\begin{aligned}
\bar{W}(S(G)) & =\frac{1}{2} \sum_{i} i \bar{D}(S(G), i) \\
& =\frac{1}{2} \sum_{i} 2 i \bar{D}(G, i)+\frac{1}{2} \sum_{i} 2 i \bar{D}(L(G), i) \\
& =2 \bar{W}(G)+2 \bar{W}(L(G)) .
\end{aligned}
$$

Therefore, $\frac{|\operatorname{Aut}(S(G))|}{|V(S(G))|} \widehat{W}(S(G))=\frac{2|\operatorname{Aut}(G)|}{|V(G)|} \widehat{W}(G)+\frac{2|\operatorname{Aut}(L(G))|}{|V(L(G))|} \widehat{W}(L(G))$, which completes our proof.

Lemma 2.5. Suppose $G$ is a tree with at least three vertices. Then $\operatorname{Aut}(T(G)) \cong \operatorname{Aut}(G)$.

Proof. The map $\Phi: \operatorname{Aut}(G) \longrightarrow \operatorname{Aut}(T(G))$ defined in a similar way as Lemma 2.3, is an isomorphism. Since if $\alpha \in \operatorname{Aut}(G)$ then $\Phi(\alpha) \in \operatorname{Aut}(T(G))$ and for $\beta \in \operatorname{Aut}(T(G))$ we have $\alpha=\left.\beta\right|_{G} \in \operatorname{Aut}(G)$, as desired.

Theorem 2.6. With hypothesis of Lemma 2.5, $\widehat{W}(T(G))=\frac{2 n-1}{n} \widehat{W}(G)+\frac{2 n-1}{n-1} \widehat{W}(L(G))$.

Proof. Suppose $x$ and $y$ are vertices of $T(G)$ such that $x \approx_{T(G)} y$ and $d_{T(G)}(x, y)=k$. If $x, y \in G$ then we claim that $d_{G}(x, y)=k$. To prove, we first notice that $G$ is a subgraph of $T(G)$. Next we assume that $P: x=u_{0}, u_{1}, \ldots, u_{h}=y$ is the unique path in $G$ 
and $P^{\prime}: x=v_{0}, v_{1}, \ldots, v_{k}=y$ is a shortest path in $L(G)$ connecting $x$ and $y$. If $v_{1}$ is a vertex in $L(G)$ and $v_{2}$ is a vertex in $G$ then by interchanging $v_{0}, v_{1}, v_{2}$ by $v_{0}, v_{2}$ we obtain another path $P^{\prime \prime}$ in $L(G)$ such that $l\left(P^{\prime \prime}\right)<l\left(P^{\prime}\right)$, a contradiction. Thus, if $\left.v_{1} \in V(L(G))\right)$ then $v_{2}, v_{3}, \ldots, v_{k-1} \in V(L(G))$ and $l(P)<l\left(P^{\prime}\right)$. This shows that $v_{1} \notin V(L(G))$. By continuing this method, one can see that all vertices of $P^{\prime}$ are in vertices of $G$. Therefore, $P=P^{\prime}$. A similar argument shows that in other case that $x$ and $y$ are corresponding to vertices in $L(G)$, a shortest path in $L(G)$ and $T(G)$ will be the same and so $\bar{W}(T(G))=\bar{W}(G)+\bar{W}(L(G))$. Therefore, $\frac{|\operatorname{Aut}(T(G))|}{|V(T(G))|} \widehat{W}(T(G))=$ $\frac{|\Gamma|}{|V(G)|} \widehat{W}(G)+\frac{|\operatorname{Aut}(L(G))|}{|V(L(G))|} \widehat{W}(L(G))$, which completes the proof.

Lemma 2.7. Suppose $G$ is a tree with at least three vertices. Then $\operatorname{Aut}(Q(G)) \cong \operatorname{Aut}(G)$.

Proof. Since $Q(G)=L(G) \cup S(G)$, Lemmas 2.1, 2.3 and a similar argument as Lemma 2.3 completes the proof.

Theorem 2.8. With hypothesis of Lemma 2.7, $\widehat{W}(Q(G))=\frac{4 n-2}{n} \widehat{W}(G)$.

Proof. Suppose $x$ and $y$ are vertices of $Q(G)$ such that $x \approx_{Q(G)} y$. If $x, y$ are corresponding to the vertices of $L(G)$ then $d_{Q(G)}(x, y)=d_{L(G)}(x, y)$. Suppose $x, y$ are vertices of $G$ with distance $k$ and $P: x=u_{0}, u_{1}, \ldots, u_{k-1}, u_{k}=y$ is a shortest path in $G$ connecting $x$ and $y$. If $x$ and $y$ are adjacent in $G$ then distance between them in $Q(G)$ will be 2 . In other case, the path $P^{\prime}: x=u_{0}, e_{1}, \ldots, e_{k}, u_{k}=y$ has length $k+1$, where $e_{i}=u_{i-1} u_{i}, 1 \leq i \leq k$. In the case that $x, y \in L(G)$, the sum of distances is $\sum_{i} i \bar{D}(L(G), i)$ and in the second case the summation will be $\sum_{i} \bar{D}(G, i)+\sum_{i} \widehat{D}(G, i)$. Then we have $\bar{W}(Q(G))=\bar{W}(G)+\frac{1}{2} \sum_{i} \widehat{D}(G, i)+\bar{W}(L(G))$. By applying Theorem 1 , the result is obtained.

Lemma 2.9. Suppose $G$ is a tree with at least three vertices. Then $\operatorname{Aut}(G)$ is isomorphic to a proper subgroup of $\operatorname{Aut}(R(G))$.

Proof. It is easy to see that the mapping $\Phi: \operatorname{Aut}(G) \longrightarrow \operatorname{Aut}(R(G))$ given by the same definition as Lemma 2.3 is a one-to-one homomorphism, as desired. Since $G$ is a tree, it has at least a pendant vertex and so $R(G)$ has an automorphism of order 2 in $\operatorname{Aut}(R(G)) \backslash$ $\Phi(\operatorname{Aut}(G))$, proving the lemma.

Theorem 2.10. With hypothesis of Lemma 2.9, $\widehat{W}(R(G))>\frac{4 n-2}{n} \frac{|\operatorname{Aut}(G)|}{|\operatorname{Aut}(R(G))|} \widehat{W}(G)$.

Proof. Suppose $x$ and $y$ are vertices in $R(G)$. Similar to Theorem 2.8, if $x, y \in G$ then $d_{R(G)}(x, y)=d_{G}(x, y)$. In this case, the sum of distances is at least $\sum_{i} i \bar{D}(G, i)$. If $x$ and $y$ are corresponding to vertices $w$ and $z$ of $L(G)$ such that $d_{L(G)}(w, z)=k$ then $d_{R(G)}(x, y)=k+1$ and so the sum of distances is at least $\sum_{i} i \bar{D}(L(G), i)+\sum_{i} \widehat{D}(G, i)$.

Now, we assume that $u$ and $v$ are two pendants in $G$ and in the same orbits under the action of $\operatorname{Aut}(G)$ on vertices and $e$ and $f$ are edges such that $u$ is incident to $e$ and $f$ is incident to $v$. Then $e$ and $f$ are in the same orbits under the action of $\operatorname{Aut}(G)$ on edges. Hence, in $R(G)$, all elements of $\{u, v, e, f\}$ are in the same orbits under the action of $\operatorname{Aut}(R(G))$. Since, corresponding to $d_{G}(u, v)$ in $\bar{W}(G)$ there exists at least a quantity in the form of $d_{R(G)}(u, v)+d_{R(G)}(e, f)+d_{R(G)}(u, f)+d_{R(G)}(v, e)+d_{R(G)}(u, e)+$ 
$d_{R(G)}(v, f)$ in $\bar{W}(R(G))$, by Theorem $1, \bar{W}(R(G))>2 \bar{W}(G)$, which completes our argument.

\section{Examples}

In this section, we apply our results in the pervious section. We denote the cyclic group of order $n$ by $\mathbb{Z}_{n}$ and the symmetric group on $n$ symbols by $\operatorname{Sym}(n)$. We notice that by Lemmas 2.1-2.7, if $G$ is a tree with at least three vertices then

$$
\operatorname{Aut}(G) \cong \operatorname{Aut}(L(G)) \cong \operatorname{Aut}(S(G)) \cong \operatorname{Aut}(T(G)) \cong \operatorname{Aut}(Q(G)),
$$

and also by Lemma 2.9, $\operatorname{Aut}(G) \leq \operatorname{Aut}(R(G))$.

Example 3.1. In this example, $\widehat{W}\left(P_{n}\right), \widehat{W}\left(L\left(P_{n}\right)\right), \widehat{W}\left(S\left(P_{n}\right)\right), \widehat{W}\left(T\left(P_{n}\right)\right), \widehat{W}\left(Q\left(P_{n}\right)\right)$ and $\widehat{W}\left(R\left(P_{n}\right)\right)$ are calculated where $P_{n}$ is a path with $n$ vertices. To do this, we assume that $V\left(P_{n}\right)=\left\{v_{i}\right\}_{i=1}^{n}$ and $E\left(P_{n}\right)=\left\{e_{i}=v_{i} v_{i+1}\right\}_{i=1}^{n-1}$. We first notice that the automorphism group of $P_{n}$ is generated by an element $\alpha$ of order 2 , where

$$
\alpha= \begin{cases}\left(v_{1}, v_{n}\right)\left(v_{2}, v_{n-1}\right) \ldots\left(v_{\frac{n}{2}}, v_{\frac{n+2}{2}}\right) & n \text { is even } \\ \left(v_{1}, v_{n}\right)\left(v_{2}, v_{n-1}\right) \ldots\left(v_{\frac{n-1}{2}}, v_{\frac{n+1}{2}}\right) & n \text { is odd }\end{cases}
$$

Therefore, $\operatorname{Aut}\left(P_{n}\right) \cong \mathbb{Z}_{2}$. The modified Wiener index of $P_{n}$ was computed in [3, Example 5.6] as

$$
\widehat{W}\left(P_{n}\right)= \begin{cases}\frac{n^{3}}{8} & n \text { is even } \\ \frac{n^{3}-n}{8} & n \text { is odd }\end{cases}
$$

On the other hand, if $n$ is even, then

$$
\bar{D}\left(P_{n}, i\right)= \begin{cases}0 & i \text { is even } \\ 2 & i \text { is odd }\end{cases}
$$

and if $n$ is odd, then

$$
\bar{D}\left(P_{n}, i\right)= \begin{cases}2 & i \text { is even } \\ 0 & i \text { is odd }\end{cases}
$$

Therefore,

$$
\sum_{i=1}^{n-1} \bar{D}\left(P_{n}, i\right)= \begin{cases}n & n \text { is even } \\ n-1 & n \text { is odd }\end{cases}
$$


By applying Theorems 2.2-2.10, we obtained the following equations,

$$
\begin{aligned}
& \widehat{W}\left(L\left(P_{n}\right)\right)= \begin{cases}\frac{n^{3}-3 n^{2}+2 n}{8} & n \text { is even } \\
\frac{(n-1)^{3}}{8} & n \text { is odd, }\end{cases} \\
& \widehat{W}\left(S\left(P_{n}\right)\right)=\frac{2 n^{3}-3 n^{2}+n}{4}, \\
& \widehat{W}\left(T\left(P_{n}\right)\right)=\frac{2 n^{3}-3 n^{2}+n}{4}, \\
& \widehat{W}\left(Q\left(P_{n}\right)\right)= \begin{cases}\frac{2 n^{3}-n^{2}}{4} & n \text { is even } \\
\frac{2 n^{3}-n^{2}-2 n+1}{4} & n \text { is odd, }\end{cases} \\
& \widehat{W}\left(R\left(P_{n}\right)\right)= \begin{cases}\frac{2 n^{3}-n^{2}}{4} & n \text { is even } \\
\frac{2 n^{3}-n^{2}+2 n-1}{4} & \text { is odd }\end{cases}
\end{aligned}
$$

For the last equality, we notice that the automorphism group of $R\left(P_{n}\right)$ can be generated by three elements $\alpha, \beta$ and $\gamma$ as follows:

$$
\begin{aligned}
& \alpha=\left(v_{1}, e_{1}\right), \\
& \beta=\left(v_{n}, e_{n}\right) \text {, } \\
& \gamma= \begin{cases}\left(v_{1}, v_{n}\right)\left(v_{2}, v_{n-1}\right) \ldots\left(v_{\frac{n}{2}}, v_{\frac{n}{2}+1}\right)\left(e_{1}, e_{n-1}\right)\left(e_{2}, e_{n-2}\right) \ldots\left(e_{\frac{n}{2}-1}, e_{\frac{n}{2}+1}\right) & n \text { is even } \\
\left(v_{1}, v_{n}\right)\left(v_{2}, v_{n-1}\right) \ldots\left(v_{\frac{n-1}{2}}, v_{\frac{n+3}{2}}\right)\left(e_{1}, e_{n-1}\right)\left(e_{2}, e_{n-2}\right) \ldots\left(e_{\frac{n+1}{2}}, e_{\frac{n+3}{2}}\right) & n \text { is odd. }\end{cases}
\end{aligned}
$$

It is easy to see that this group is isomorphic to the dihedral group $D_{8}$.

In the following example to compute the modified Wiener index, we apply the concept of semidirect product and wreath product of groups together with our results in last section. Let $G$ be a group with a subgroup $H$ and a normal subgroup $N$ such that $G=H N$ and $H \cap N=1$. Then $G$ is called the semidirect product of $N$ by $H$. To define the notion of wreath product, we assume that $A$ and $H$ are groups, $X$ is a set and $H$ acts on $X$. Define $K=\prod_{w \in X} A_{w}$, where $A_{w} \cong A$. If we consider the elements of $K$ as arbitrary sequences of elements of $A$ with componentwise multiplication then the action of $H$ on $X$ can be extended in a natural way to an action of $H$ on the group $K$ by $h\left(a_{\omega}\right)=\left(a_{h^{-1}} \omega\right)$. Then the wreath product $A$ ? $H$ of $A$ by $H$ is the semidirect product $H$ by $K$.

Example 3.2. Suppose $S_{n}$ is an star with a vertex of degree $n$ and $n$ pendant vertices. Since $S_{n}$ has exactly one vertex of degree $n$, this vertex will be fixed under each automorphism of $S_{n}$. On the other hand, all pendants can be imaged under permutations to each other. Thus $\operatorname{Aut}\left(S_{n}\right) \cong \operatorname{Sym}(n)$. According to [3, Example 5.8], the modified Wiener index of $S_{n}$ with $n+1$ vertices is equal to

$$
\widehat{W}\left(S_{n}\right)=n^{2}-1
$$

Also,

$$
\bar{D}\left(S_{n}, i\right)= \begin{cases}0 & i=1 \\ (n-1) n ! & i=2 .\end{cases}
$$

Therefore, by applying Theorems 2.2-2.10, 


$$
\begin{aligned}
& \widehat{W}\left(L\left(S_{n}\right)\right)=\frac{n^{2}-n}{2}, \\
& \widehat{W}\left(S\left(S_{n}\right)\right)=5 n^{2}-3 n-3, \\
& \widehat{W}\left(T\left(S_{n}\right)\right)=\frac{6 n^{2}-3 n-3}{2}, \\
& \widehat{W}\left(Q\left(S_{n}\right)\right)=4 n^{2}-2 n-2, \\
& \widehat{W}\left(R\left(S_{n}\right)\right)=\frac{8 n^{2}-2 n-3}{2} .
\end{aligned}
$$

For the proof of last equality, we assume that

$$
V\left(S_{n}\right)=\left\{v_{0}, v_{1}, v_{2}, \ldots, v_{n}\right\} \text { and } E\left(S_{n}\right)=\left\{e_{i}=v_{0} v_{i} \mid 1 \leq i \leq n\right\} .
$$

The automorphism group of $R\left(S_{n}\right)$ has automorphisms $\tau_{i}$ such that $\tau_{i}: v_{i} \mapsto e_{i}$ and $\tau_{i}$ fixes other vertices of the graph. Suppose $A_{i}=\left\langle\tau_{i}\right\rangle$. Then $A_{i} \cong \mathbb{Z}_{2}$ and

$$
K=\underbrace{\mathbb{Z}_{2} \times \ldots \times \mathbb{Z}_{2}}_{n \text { times }}
$$

is isomorphic to a subgroup of $\operatorname{Aut}\left(R\left(S_{n}\right)\right)$. On the other hand, $\operatorname{Sym}(n)$ acts on $K$ by $\alpha\left(a_{i}\right)=\left(a_{\alpha^{-1} i}\right)$. Hence $\operatorname{Aut}\left(R\left(S_{n}\right)\right) \cong \mathbb{Z}_{2}$ 々 $\operatorname{Sym}(n)$.

Acknowledgements. We are indebted to anonymous referees for their suggestions and helpful remarks. The research of the authors are partially supported by the University of Kashan under grant no. 364988/88.

\section{References}

[1] A. A. Dobrynin, R. Entringer and I. Gutman, Wiener index of trees: Theory and applications, Acta Appl. Math. 66 (2001), 211-249.

[2] A. A. Dobrynin, I. Gutman, S. Klavžar and P. Žigert, Wiener index of hexagonal systems, Acta Appl. Math. 72 (2002), 247-294.

[3] A. Graovac and T. Pisanski, On the Wiener index of a graph, J. Math. Chem. 8 (1991), 53-62.

[4] I. Gutman and L. S̆oltés, The range of the Wiener index and its mean isomer degeneracy, $Z$. Naturforsch. 46a (1991), 865-868.

[5] W. Imrich and S. Klavžar, Product Graphs: Structure and Recognition, Wiley, New York, USA, 2000.

[6] F. Koorepazan-Moftakhar and A. R. Ashrafi, Distance under Symmetry, MATCH Commun. Math. Comput. Chem. 74 (2015), 259-272.

[7] J. Lauri and R. Scapellato, Topics in Graph Automorphisms and Reconstruction, Cambridge University Press, Cambridge, 2003.

[8] W. Yan, B.-Y. Yang and Y.-N. Yeh, The behavior of Wiener indices and polynomials of graphs under five graph decorations, Appl. Math. Lett. 20 (2007), 290-295.

[9] H. J. Wiener, Structural determination of paraffin boiling points, J. Am. Chem. Soc. 69 (1947), $17-20$. 